Physical Sciences | Dr Žiga Gosar

\title{
Improving prospects for polymer surface coatings
}

\section{Within the automotive} light industry, aluminium evaporated on plastic high gloss reflector surfaces. From a quality point of view and this surface must retain its luminance and reflectiveness for 15 years. However, the non-treated metal parts oxidise, meaning that it loses its luminance and reflectiveness. For this reason, it is protected with silicon oxide or a glasslike protection layer. Glassformed in high vacuum plas polymerisation process. Dr Žiga Gosar. Head of R\&D at ELVEZ Slovenia explores advanced new ways to overcome thes barriers. Named 'plasmaenhanced chemical vapour deposition' (PECVD), his team technique uses plasma to both roughen polymer surfaces groups, all on industrial scales. ade up of long chains of

organic molecules, polymers wide variety of products we use in our everyday lives. In car manufacturing, for example, many important components are made by printing and painting polymer surfaces, coating them with metals, and treating them with other protective coatings. However, it is difficult for manufacturers to do this effectively using cheaper, more widely available polymers, owing to their water-resisting, or 'hydrophobic' properties, which make them less to paints and coatings.

The goal is to make hydrophilic surfaces. The problem can be reduced through the technique of 'functionalisation, which attaches oxygen-rich groups of molecules, surfaces. If prepars," to polymer groups can bind more strongly the molecules in paints and cotings, making surfaces more adhesive overal. Since functionalisation traditionally involves the use of harmful chemicals, manufacturers have recently begun to reduce their environmental impact by using plasma - a state of matter containing hot, freely-flowing charged Unfortunately, despite the advantages of this approach, many types of functionalise using current plasm based techniques. In addition, many polymers which can be treated in this way will suffer from deterioration over time, lowering the quality of manufacturers' final products. In Slovenia, where the economy is strongly reliant on the automotive industry, problem. However, Dr Ziga Gosar of ELVEZ Slovenia and his colleagues believe that a more all-encompassing

\section{SIDE-EFFECTS OF}

(n)

To create the plasma required for typically heat up gases in a rear containing mixtures of atoms including argon and oxygen. To form functional groups, these oxygen atoms will be ionised and excited to higher energ levels, making it easier for them to these shortcomings are a significant attach to polymer surfaces. For the process po succed, reses. plasma discharged from the reactor producing it If this are set correctly coating and painting finishes will easily adhere to polymer molecules.

In addition to functionalising polymers, his technique has the ability to etch dips and valleys into polymer surfac This is a desirable effect, however, until recently the inter plasma and polymer which led to this effect were poorly understood Than to recent discoveries, we now know that the etching is non-uniform, due to inherent the fluctuations inherently displayed by plasma on molecular scales. Therefore, it can result in ches With the rediation py trealing surfaces Wher its ionisation source is removed known as its 'afterglow'. However, a growing body of research is now exploring how nanoscale etching could be exploited to increase the durability and adhesiveness of polymer surfaces,

\section{PROBING THE PROPERTIES}

OF PLASMA ETCHING

In recent years, techniques for creating higher-pressure plasma discharges have enabled researchers to produce high volumes of uniform plasma and sustain them for long periods of time. Naturally, this has led to a huge variety functios into how plasma induced industrial scales but mach other on aso used the advance to explore the characteristics of plasma etching for the first time. Several groups, for example, have investigated what happens when small patches of polymer surfaces are coated with electrode materials, protecting them from the effects of plasma exposure. Intriguingly, this causes columns of fibre-like nanostructures to form.

Materials physicists soon realised that these roughened surfaces could bind to paints and coatings far more effectively than smooth surfaces, solving the adhesiveness problem faced by industries today. Leading on from have probed the durability of fibrous

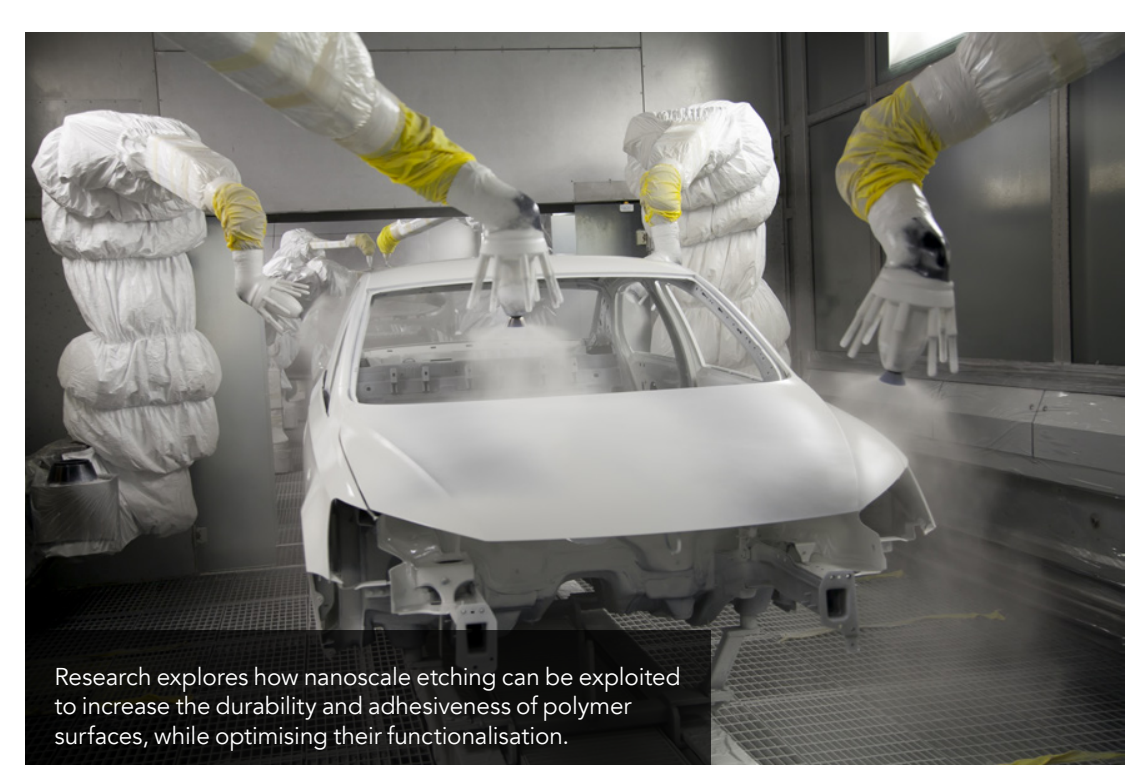

polymer nanostructures. They found $\begin{array}{ll}\text { polymer nanostructures. They found } & \text { of nanostructure like granules can } \\ \text { that the columns can easily collapse } & \text { also form. Overall, these previous }\end{array}$ studies seem to point to an ideal treatment times.

Further studies have explored how etched nanostructures are influenced by factors including the compositions of plasmer themselves, and the pow of around 10 are to be fully enjoyed. Unfortunang this is far too long for the process to become practical for industrial use. However, Gosar points out that as a whole, previous studies have almost completely neglected to consider the on these characteristics, other types of the plasma itself.

Gosar has uncovered sophisticated new ways to ensure that functionalised polymer surfaces are strongly adhesive and remain durable over time.

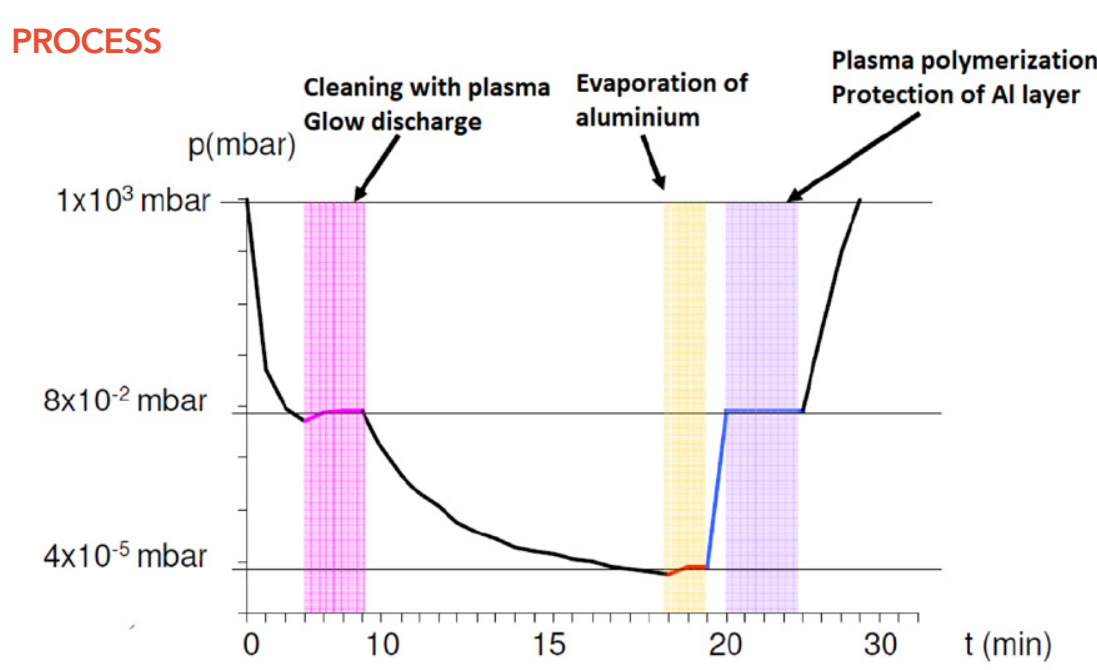


CHARACTERISING POLYMER-

SMA INTERACTIONS

Their research, Gosar and his

their deeper investigations objectives

functionalisation. The first was is to

study how etching onto specific polymer

surfaces is influenced by different types

of reactive oxygen species contained

within the plasma. Their ultimate

aim was to use estimations of the

plasma-related mechanisms leading

to nanostructure formation, including

the density of positive ions and neutra

atoms, to achieve optimal etching

nd functionalisation with reasonable

reatment times.

Thirdly, the researchers aimed to

which would give rise to optinal parameters Finally, they would study how these

parameters could be upsized to plasm a reactors with volumes on scales of cubic metres. Ultimately, Gosar's team hopes that these efforts will allow them to achieve fast, energy-efficient plasma functionalisation, suitable for industria applications, for the first time.

\section{DEPOSITION ON}

NDUSTRIAL SCALES

In one 2019 study, Gosar and

colleagues used a plasma reactor with

a volume of $5 \mathrm{~m}^{3}$ to implement their

new technique of "plasma-enhanced As a more advanced form of the already well-established technique f chemical vapour deposition, this mixture of oxygen and the molecule hexamethyldisiloxane (HMDSO). The team then optimised discharge parameters of the plasma, by monitoring it using 'residual gas mass spectrometry' and 'optical emission spectroscopy'; the adjusting its parameters accordingly.

Once optimised, Gosar's team exposed the plasma to a polymer surfaced roughened by previous etching. They then treated the now functionalised surface both with aluminium, and a protective coating containing characteristics of the resulting treated surface, using the combing treated

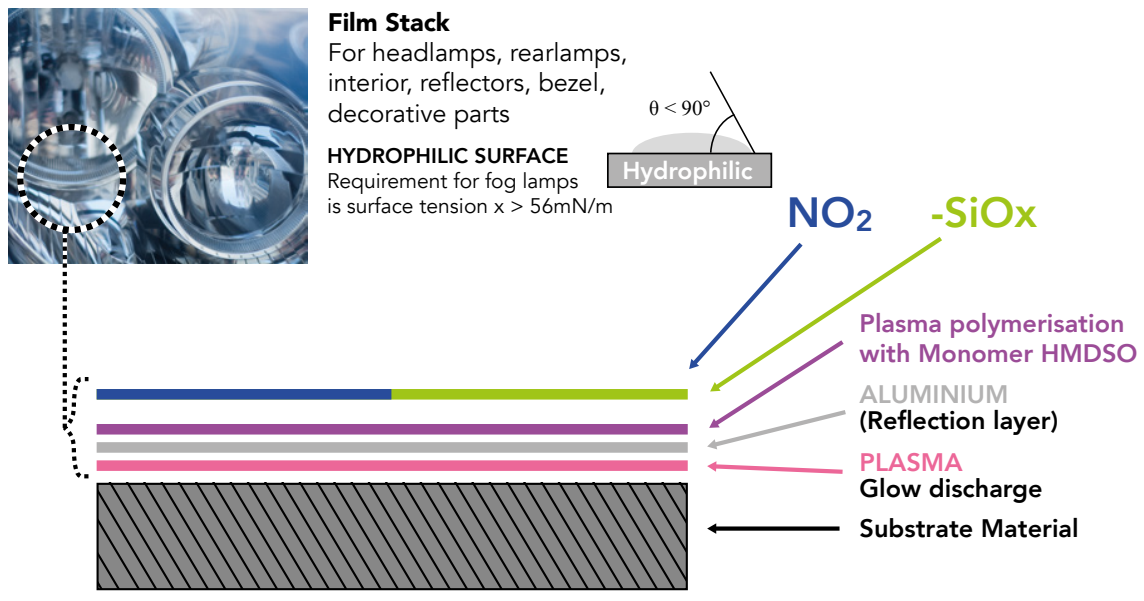

Named 'plasma-enhanced chemical vapour deposition' (PECVD), the technique uses plasma to both roughen polymer surfaces and introduce new molecular groups.

of 'secondary ion mass spectrometry' at different flow rates, to suit the (SIMS) and 'x-ray photoelectron spectroscopy' (XPS). This allowed the team to determine the composition and textures of the surfaces on nanoscales, affording them an in-dept understanding of how plasma and protective coatings interact with polym
surfaces inside industrial-scale reactors as PECVD unfolds.

\section{FURTHER EXPLORATIONS}

\section{OF PECVD}

precisely quantifies, the researchers of their innovative PECVD technique, including plasma density, flow intensity, and reactor pumping speed. Applying similar analytical techniques to their previous study, Gosar and colleagues were able to monitor how their plasma behaved in different discharge conditions, meaning optimal parameters cost tecing only the simple, lowspectroscopy (OES).

In addition, the team explored how HMDSO gas could be introduced applications. Their mula ments showed that PECVD allows coating deposition rates and plasma ion densities to remain almost perfectly constant over time; increasing linearly with flow rate. Together, these studies have clearly demonstrated the advantages of PECVD over previous approach

\section{MPROVING MANUFACTURING}

\section{TECHNIQUES}

discoveries, Gosar functionalised polymer surfaces are strongly adhesive, and remain durable over time. By ensuring that plasma re sufficient on industria scales to allow uniform deposition by grould resolve the issues faced by groups including Slovenia's car manufacturers. The team will now continue to improve their techniques to be even better suited to practical rew oting to manterias for research in and his colleagues have uncovered sophisticated new ways to ensure that

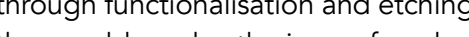
applications, and may even open up

\section{Behind the Research}

Dr Žiga Gosar

E: ziga.gosar@gmail.com T: 0038641949840

Research Objectives

Ziga Gosar's work is focused on the optimisation of the production processes phase of plasma polymerisation.

\section{Detail}

Žiga Gosar

Ulica Antona Tomsisca 35

294 Visnja Gora, Slovenia, EU

Dr Žiga Gosar (M), received his diploma degree mechanical engicering in 2010 and his $P$ D in 2014, during which time he applied the high vacuum metallisation process within EIVEZ. He has been Head of Research \& Development at ELVEZ since 2014. Dr Gosar received a Rectors Reward for Innovation in 2017. He is currently working towards his second PhD (plasma polymerisation) at the International Postgraduate school of Jožef Stefan. He is a member of SRIP ToP, and coordinator for robotics, and on the advisory board for project Co-production CeLL performing Human-Robot Collaborative AssEmbly.

\section{Collaborators}

- Simona Petrić (CEO of ELVEZ)

- Prof DrMran Mozetí (mentor)

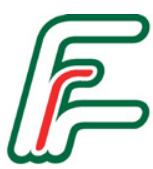

Doc Dr Rok Zaplotnik (co-mentor)

\section{References}

Gosar, Ž., Kovač, J., Mozetič, M., Primc, G., Vesel, A. and Zaplotnik, R. (2019). Deposition of SiOxCyHz Protective Coatings on Polymer Substrates in an Industrial-Scale coatings9040234

Gosar, Ž., Kovač, J., Mozetič, M., Primc, G. and Vesel, A. (2019). Characterization of Gaseous Plasma Sustained in Mixtures of HMDSO and $\mathrm{O} 2$ in an Industrial-Scale Reactor. Plasma Chemistry and Plasma Processing, pp.18. doi:10.1007/s11090-019-10026-5

Gosar, Ž., Đonlagić, D., Pevec, S., Kovač, J., Mozetič, M Primc, G., Vesel, A. and Zaplotnik, R. (2019). Deposition Reactor. Materials, 12(19) P. 3238 , do: $103390 /$ ma12193238

\section{Personal Response}

\section{What is next for your research?}

1. Innovative sensors for real-time monitoring of deposition rates in plasma-enhanced chemical vapour deposition
(PECVD) systems. This is an applied research project. A multidisciplinary team with expertise in sensors, optics masma sensor for real-time monitoring of deposition rates in plasma-enhanced chemical vapour deposition (PECVD) systems, in particular for depositing thin silica layers on polymer substrates. The sensor will be superior as friendly and muact cheaper, so it will be suitable for

applyation in industry. particularly in automotive ind sty. application in industry, particularly in automotive industry.
The know-how gained within the project will be protected by a patent application. If successful, the project will suitable for trading.

2. Initial stages in surface functionalis tion o polym 2. Initial stages in surface functionalisation of polymers
by plasma radicals. Although thousands of scientific papers have been published on interaction between non-equilibrium gaseous plasma and polymers, we have not found a single paper on experimental determination of initial stages of surface functionalisation. The crientific challenge addressed in this project is therefor formed initially upon exposure of selected polymers to selected reactive gaseous species, and the fluence of said species necessary to saturate the polymer surface with a specific functional group. We shall develop a dedicated chamber which will enable adjustabler preparation reactive species in the range between $10^{16}$ and $10^{20} \mathrm{~m}$ for in-situ treatment of a polymer, probes capable of realtime measuring of the densities of reactive species, and our high-resolution XPS for surface characterisation. The correspond a fluences (published as a monoups and and several scientific papers. Although only basic research is foreseen, the results will be suitable for users of plasma technology who often over-treat their materials, especially when using gaseous plasma for treatment of polymeric body implants, materials for food Sakaging and sinilar applications.

components by gaseous plasma.
4. Plasma glow plug for car engines.

5. Innovative methods for purification of wastewater. 